\title{
CONTROL INTERNO EN EL DESEMPEÑO DOCENTE Y SU RELACIÓN CON EL LOGRO DE OBJETIVOS INSTITUCIONALES. CASO: UNIVERSIDAD TÉCNICA DE MANABÍ
}

\author{
INTERNAL CONTROL IN TEACHING PERFORMANCE AND ITS RELATIONSHIP \\ WITH THE ACHIEVEMENT OF INSTITUTIONAL OBJECTIVES. CASE: \\ UNIVERSIDAD TÉCNICA DE MANABÍ
}
Gladys Varinia Salazar Cobeña Universidad Técnica de Manabí Portoviejo, Ecuador ORCID: http://orcid.org/0000-0002-7889-9111 Correo electrónico: variniasalcob@hotmail.com

\section{RESUMEN}

Objetivo: Esta investigación tuvo como objetivo determinar el control interno en el desempeño docente y su relación con el logro de los objetivos institucionales de la Universidad Técnica de Manabí. Método: El enfoque del estudio es cuantitativo, el tipo de investigación descriptiva, correlacional y exploratoria, el diseño transeccional no experimental. Se aplicó un cuestionario en escala de Likert con un coeficiente de confiabilidad Alfa de Cronbach de 0,81, a 216 docentes de las facultades e institutos que imparten asignaturas a todas las carreras de la Universidad. Se recopilaron los resultados de la evaluación integral de desempeño docente del período 2017-2018 generados por el Sistema de Gestión Académica y se categorizaron los niveles de desempeño conforme al Reglamento de Evaluación Integral al Desempeño del Personal Académico de la Universidad Técnica de Manabí de 2017. Resultados: Los resultados revelaron que la mayoría de las evaluaciones se ubicaron en la categoría más alta y que no existe correlación estadística entre el control interno en el desempeño del talento humano docente y el logro de objetivos institucionales. Conclusiones: Se concluye que el nivel de control interno en el desempeño docente no contribuye al logro de los objetivos institucionales, producto de que la universidad no tiene un sistema de control interno formal para supervisar el cumplimiento de las funciones sustantivas de la educación superior.

Palabras clave: Evaluación; desempeño; objetivos; control; docente; universidad.

\begin{abstract}
Objective: This research aimed to determine internal control in teaching performance and its relationship with the achievement of the institutional objectives of the Universidad Técnica de Manabí. Method: The focus of the study is quantitative, the type was a descriptive, correlational and exploratory research, with a non-experimental cross-sectional design. A Likert scale questionnaire with a Cronbach Alpha reliability coefficient of 0.81 was applied to 216 teachers of the faculties and institutes that teach subjects to all the University degree courses. The results of the comprehensive evaluation of the teaching performance of the 2017-2018 period generated by the Academic Management System were compiled and the performance levels were categorized according to the Regulation of Integral Evaluation of the Academic Personnel Performance of the Technical University of Manabi of 2017. Results: The results revealed that the majority of the evaluations were in the highest category and that there is a direct statistical correlation between the level of performance of the teaching human talent and the achievement of institutional objectives. Conclusions: It is concluded that the level of internal control in teacher performance does not contribute to the achievement of institutional objectives, as a result of the university not having a formal internal control system to supervise the fulfillment of the substantive functions of higher education.
\end{abstract}

Keywords: Evaluation; objectives; performance; control; teacher; university. 


\section{INTRODUCCIÓN}

A partir de la Constitución de la República del Ecuador promulgada en octubre de 2008, el país ha pasado por una transición legal en lo que a sistema de educación superior se refiere, instaurándose una estructura universitaria regida por Asamblea Nacional Constituyente (2008) que refiere "los principios de autonomía responsable, cogobierno, igualdad de oportunidades, calidad, pertinencia, integralidad y autodeterminación para la producción del pensamiento y conocimiento en el marco del diálogo de saberes, pensamiento universal y producción científica tecnológica global" (p.163). Las acciones de regulación y control de la educación superior son de absoluta competencia del Estado ecuatoriano, por ende, el sistema de educación superior debe articularse al sistema de educación nacional y al plan de desarrollo de la nación.

La Ley Orgánica de Educación Superior (LOES) emitida en 2010 incluyó aspectos fundamentales como los organismos que integran y rigen la educación superior en el Ecuador, la pertinencia y calidad educativa, la evaluación y acreditación universitaria y el escalafón del personal académico de universidades y escuelas politécnicas públicas y privadas, mismos que se mantienen en la LOES de 2018. El Consejo de Educación Superior (CES) (2018) establece como requisito para la permanencia, promoción, retención y estímulos del docente universitario, la evaluación integral del desempeño, a partir de la evaluación del cumplimiento de las funciones sustantivas de la educación superior en el Ecuador. Ante este hecho, el CES (2018) determina que "la evaluación integral del desempeño se aplicará a todo el personal académico de las instituciones de educación superior, públicas y particulares. La evaluación integral de desempeño abarca las actividades de docencia, investigación y dirección o gestión académica” (p. 46).

Ante ello, las universidades y escuelas politécnicas del Ecuador evalúan periódicamente el desempeño de su talento humano docente. La Universidad Técnica de Manabí (UTM) realiza evaluaciones integrales de desempeño desde 2012 como un mecanismo de gestión para alcanzar los objetivos institucionales. A través de los resultados de estas evaluaciones, la Universidad pretende mejorar el rendimiento laboral de los docentes, alcanzar su excelencia y calidad académica, permanecer en el tiempo, y conservar su reconocimiento y prestigio institucional.

Chiavenato (2017) define a la evaluación de desempeño como "una apreciación sistémica de cómo se desempeña una persona en un puesto y de su potencial de desarrollo" (p. 210). Según Alles (2019), "la evaluación de desempeño es una herramienta fundamental entre las buenas prácticas de Recursos Humanos y se relaciona con otros subsistemas" (p. 311). Antes de iniciar un proceso de eva- luación de desempeño del personal es necesario establecer componentes, actores e instrumentos que formarán parte de la evaluación, analizar las características del puesto que ocupa el colaborador, e informar a los actores de la evaluación el alcance y objetivos propuestos en el período a evaluar. Weihrich, Cannice y Koontz (2017) sostienen que “...al evaluar el desempeño, los sistemas de evaluación que consideran objetivos cuantificables preestablecidos tienen un valor extraordinario" (p. 334).

Dessler y Varela (2017) exponen que las competencias y estándares de conducta son formas de evaluación de desempeño que "consisten en evaluar a los trabajadores con base al dominio de competencias (por lo general, las habilidades, el conocimiento y las conductas personales) que se requieren al realizar el trabajo" (p. 187). Las organizaciones emplean diferentes enfoques para evaluar el rendimiento de sus colaboradores, entre los principales están las evaluaciones verticales de 90 , de 180 , de 270 y de 360 grados. Esta última es un subsistema de talento humano integrado por múltiples evaluadores, en el que participan los propios evaluados (autoevaluación), sus superiores, subordinados, compañeros, pares y, en algunas ocasiones, los clientes internos y/o externos (Bohlander, Snell y Morris, 2018). La función del evaluador es emitir juicios de valor respecto del desempeño de sus colaboradores basándose en criterios preestablecidos, tomando como base la descripción y el análisis del control estratégico, el control de la eficacia y el logro de los objetivos institucionales, el control operativo y un análisis detallado del control (Ronquillo, Moreira y Verdesoto, 2016).

Para Homer (2013), las universidades se desarrollan con el objetivo de satisfacer las ilimitadas necesidades de la sociedad. La historia de la educación superior en la edad medieval ha demostrado que un buen profesor está al frente de todo desarrollo universitario; es aquel que confiere prestigio y reconocimiento académico a una región a través de la transformación del conocimiento.

Los objetivos de las instituciones de educación superior guardan relación con los procesos de evaluación institucional y con los de evaluación de carreras o programas de grado y posgrado que hoy en día exigen pertinencia y calidad educativa. En ese sentido, los objetivos representan los logros o metas que se pretenden alcanzar y se constituyen en la base de la evaluación del desempeño docente en cualquier institución educativa. De ahí la importancia de que los objetivos sean alcanzables, medibles y desafiantes. Alles (2012) afirma que el término objetivos hace referencia a las metas asignadas a una persona, a alcanzar en un determinado período de tiempo, usualmente un año.

Tal y como Tejedor y Jornet (2008) sostienen la evaluación de desempeño docente debe considerar un conjunto 
de mecanismos que proporcionen información confiable sobre el grado en que los colaboradores contribuyen a la consecución de los estándares requeridos para un puesto o cargo establecido, y por ende al logro de los objetivos institucionales, lo cual contribuye al desarrollo profesional y personal del talento humano como factor clave para el desarrollo de una instancia organizativa.

Las evaluaciones múltiples representan valiosos beneficios para las organizaciones, una implementación inadecuada será sumamente inconveniente en lo que respecta a la confiabilidad y credibilidad de los resultados de la evaluación. $\mathrm{Al}$ respecto, Alles (2017) señala que en el caso de las evaluaciones de desempeño en que el jefe es el único evaluador, los resultados no tendrían mayor aceptación por el colaborador, pudiendo pensar que su jefe no es parcial o mantiene una predisposición negativa ante él, o en ocasiones, favoritismo por otro colaborador.

Almuiñas y Galarza (2013) manifiestan que:

Los modelos de evaluación de desempeño se operacionalizan con distintos procedimientos metodológicos, técnicas, actores y fuentes de información, al tiempo que reflexionan sobre la existencia de problemas que surgen y que afectan los resultados de la evaluación, entre los principales se destacan: Subjetivismo, resistencia al cambio, desvinculación entre el perfil del docente y la evaluación del desempeño, mala interpretación y poca claridad de los propósitos y objetivos de la evaluación, inseguridad y temor, insuficiencias en la aplicación de las técnicas y dificultades para obtener consenso sobre los criterios para evaluar el desempeño (pp. 36-37).

En el ámbito académico, Fernandes, Sotolongo y Martínez (2016) revelaron en una investigación que docentes y estudiantes priorizaron la competencia pedagógica-didáctica en la evaluación del desempeño del personal docente. Sin embargo, se evidenciaron diferencias en la percepción del orden de importancia de las competencias académicas. Los estudiantes consideraron que deben valorarse en primer lugar las competencias de extensión universitaria, luego las de gestión académica y por último las investigativas. Por su parte, los docentes priorizaron las competencias de gestión académica, las investigativas y de extensión universitaria. Esto puede deberse a que una apropiada gestión impacta directa y positivamente en la calidad de las demás funciones sustantivas en la educación superior, lo que nos lleva a plantear que la evaluación integral al desempeño al docente es un instrumento de gestión clave para impulsar la competitividad de las instituciones de educación superior a partir de la implementación de controles diseñados, ejecutados y evaluados al interior de las mismas.
Si bien la UTM está haciendo importantes esfuerzos para incorporar la cultura de la investigación, la docencia es la función sustantiva en la cual los docentes apuntan a más logros institucionales, en comparación con la investigación y gestión académica, situación que refleja el énfasis histórico que ha tenido el Estado ecuatoriano y las universidades del país en valorar la docencia por encima de la investigación.

Los controles implementados en las actividades académicas del profesor e investigador en la UTM presentan debilidades que justifican este tipo de investigaciones a fin de que la Institución optimice el uso de sus recursos, tangibles e intangibles, lo cual contribuirá al logro de la excelencia académica de forma productiva y con altos estándares de calidad. Cuando una institución de educación superior funciona con estándares de calidad, sin desperdicio de recursos y respaldada con eficientes sistemas de control interno, el Estado asegura el resguardo de su patrimonio, el cual puede reinvertir en áreas estratégicas como educación, salud y más recursos para las universidades públicas.

El objetivo de esta investigación es determinar el control interno en el desempeño docente y su relación con el logro de los objetivos institucionales de la Universidad Técnica de Manabí, con lo que se pretende comprobar si el nivel de control en el desempeño docente permite el logro de objetivos institucionales por función sustantiva en dicha institución.

Es así que surge el siguiente problema: ¿Existe relación entre el control interno en el desempeño docente y el logro de los objetivos institucionales de la Universidad Técnica de Manabí? Luego de investigar diversas fuentes de información referidos al tema podemos responder a esta interrogante con la hipótesis siguiente: el control interno en el desempeño docente tiene relación con el logro de los objetivos institucionales en la Universidad Técnica de Manabí. Se pretende sistematizar información teórica que permita abordar el estudio de la relación entre el nivel de control interno en el desempeño docente y su contribución al logro de los objetivos institucionales en universidades y escuelas politécnicas. Desde el punto de vista metodológico, se propone un procedimiento para estudiar la relación entre evaluación de desempeño docente y logro de objetivos institucionales, con un instrumento estadísticamente confiable y validado por expertos que pueda ser utilizado en otros estudios con el mismo objetivo.

\section{MATERIALES Y MÉTODOS}

Este estudio fue realizado en la Universidad Técnica de Manabí, ubicada en la ciudad de Portoviejo, provincia de Manabí, Ecuador. La investigación tuvo un enfoque cuan- 
titativo. El tipo de investigación fue correlacional, exploratoria, y de diseño transeccional no experimental. Constó de dos fases: una documental y una de campo. En la primera fase, se sistematizaron los resultados de la evaluación integral del desempeño docente realizada en el período 2017-2018 con el fin de determinar la distribución de los resultados, los cuales fueron generados del Sistema de Gestión Académica de la Institución. Los componentes de la evaluación de desempeño del docente universitario son: la autoevaluación, realizada por el docente evaluado sobre su trabajo y su desempeño académico; la coevaluación realizada por los pares académicos (compañeros) y directivos de la Universidad; y la heteroevaluación, realizada por los estudiantes respecto del proceso de aprendizaje impartido por el docente (Consejo de Educación Superior, 2018).

De acuerdo a lo establecido en el Reglamento de Carrera y Escalafón del Profesor e Investigador (Consejo de Educación Superior, 2018) y en el Reglamento de Evaluación Integral al Desempeño del Personal Académico (Universidad Técnica de Manabí, 2016), la UTM seleccionó los siguientes componentes y ponderaciones para la evaluación integral del talento humano docente del período 2017 2018: Docencia: autoevaluación 20\%, heteroevaluación: $40 \%$, coevaluación por pares académicos $20 \%$ y directivos $20 \%$; Investigación: autoevaluación 20\%, coevaluación: pares académicos $40 \%$ y directivos 40\%; y, Gestión Académi$c a$ : autoevaluación $20 \%$, heteroevaluación: $20 \%$, coevaluación por pares académicos $30 \%$ y directivos $30 \%$.

\section{RESULTADOS}

Para el análisis de los resultados finales de las evaluaciones de desempeño docente del período 2017-2018 se consideró lo estipulado en el artículo 39 del Reglamento de Evaluación Integral al Desempeño del Personal Académico de la Universidad Técnica de Manabí (2017), en la cual se establecieron criterios cuantitativos y cualitativos para determinar el nivel de control interno en el desempeño de los docentes (cualitativo) conforme a rangos (cuantitativo). Los profesores que alcanzaron de 91 a 100 puntos pertenecen a la categoría de destacados; de 81 a 90 puntos, competentes; de 60 a 80 puntos, satisfactorios; y con calificaciones menores a 60 puntos, insatisfactorios.

En la segunda fase (fase de campo), se aplicó un cuestionario en escala de Likert con 15 ítems, validado por tres expertos y con una confiabilidad Alfa de Cronbach de 0,81 a 216 docentes con dedicación a tiempo completo, tanto titulares como contratados, que pasaron al menos por un proceso de evaluación integral docente en la UTM. El método de muestreo fue probabilístico estratificado de tipo aleatorio, el cual consideró una muestra significativa de profesores que pertenecen a los 32 departamentos de las 10 facultades y 2 institutos que imparten asignaturas a las
34 carreras de la Universidad, con el fin de determinar si las funciones sustantivas (investigación, docencia y gestión académica) desempeñadas por ellos están orientadas al logro de los objetivos institucionales. Para el análisis estadístico del estudio se utilizó el paquete estadístico IBM SPSS versión 23. Las hipótesis planteadas fueron:

$\mathrm{H}_{0}$ : El control interno en el desempeño docente no se relaciona con el logro de los objetivos institucionales en la Universidad Técnica de Manabí.

$\mathrm{H}_{1}$ : El control interno en el desempeño docente se relaciona con el logro de los objetivos institucionales en la Universidad Técnica de Manabí.

Con lo expuesto se presentan los resultados sobre el nivel de control interno en el desempeño docente de la UTM, los cuales fueron obtenidos en la fase documental:

En la tabla 1 se presenta la valoración del nivel de control interno en el desempeño docente en las categorías establecidas en el Reglamento de Evaluación Integral al Desempeño del Personal Académico de la UTM (2017). Los resultados indican que el 88,43\% (191 docentes) se ubican en el nivel destacado; 9,72\% (21 docentes), competente; $1,85 \%$ (4 docentes), satisfactorio; y, el $0 \%$ insatisfactorio.

Tabla 1

Distribución de los docentes de la UTM según nivel de desempeño

\begin{tabular}{lcc}
\hline Nivel de desempeño & Frecuencia & Porcentaje \\
\hline Destacado (91-100) & 191 & $88,43 \%$ \\
Competente $(81-90)$ & 21 & $9,72 \%$ \\
Satisfactorio $(60-80)$ & 4 & $1,85 \%$ \\
Insatisfactorio $(<60)$ & 0 & $0,00 \%$ \\
\hline
\end{tabular}

Fuente: Elaboración propia (2019)

En la fase de campo se obtuvieron los siguientes resultados relacionados con el nivel de logro de objetivos institucionales por función sustantiva.

Tal como se indica en la tabla 2, la distribución porcentual del logro de objetivos institucionales en los docentes fue la siguiente: nivel moderado $48,15 \%$, nivel alto $24,54 \%$, nivel muy alto $19,44 \%$ y nivel bajo $7,87 \%$.

Se presenta en la tabla 3 el puntaje promedio de logros de objetivos institucionales discriminados por función sustantiva. Se observa que, de acuerdo a la percepción de los docentes encuestados, la subdimensión (función sustantiva) con mayor puntaje fue la de docencia con 83,80 puntos, seguido de gestión académica con 75,58 puntos, mientras que investigación presentó el menor puntaje $(69,21)$. 
Tabla 2

Distribución porcentual de la valoración del logro de objetivos institucionales de docentes UTM

\begin{tabular}{lcc}
\hline $\begin{array}{l}\text { Nivel del logro de los } \\
\text { objetivos institucionales }\end{array}$ & Docentes & Porcentaje \\
\hline Muy alto (91-100 puntos) & 42 & $19,44 \%$ \\
Alto (81-90 puntos) & 53 & $24,54 \%$ \\
Moderado (60-80 puntos) & 104 & $48,15 \%$ \\
Bajo (<60 puntos) & 17 & $7,87 \%$ \\
\hline
\end{tabular}

Fuente: Elaboración propia (2019)

Tabla 3

Puntaje promedio de las subdimensiones de la variable logro de los objetivos institucionales

\begin{tabular}{lc}
\hline $\begin{array}{l}\text { Subdimesión del logro de objetivos } \\
\text { institucionales }\end{array}$ & $\begin{array}{c}\text { Puntaje } \\
\text { promedio }\end{array}$ \\
\hline Docencia & 83,80 \\
Investigación & 69,21 \\
Gestión académica & 75,58 \\
\hline
\end{tabular}

Fuente: Elaboración propia (2019)

La tabla 4 presenta la valoración del nivel de logro de los objetivos institucionales por subdimensiones (docencia, investigación y gestión académica). Se observaron los siguientes valores para los niveles de la función sustantiva de docencia: $37,50 \%$ (nivel muy alto); $27,78 \%$ (nivel alto); $31,02 \%$ (nivel moderado) y 3,70\% (nivel bajo). Para la subdimensión gestión académica, los valores por niveles fueron los siguientes: $21,30 \%$ (muy alto); 22,22\% (alto); $37,50 \%$ (moderado) y $18,98 \%$ (bajo). En la función investigación se observan los siguientes porcentajes por niveles: $15,28 \%$ (muy alto); $16,20 \%$ (alto); 38,43\% (moderado) y $30,09 \%$ (bajo). Estos resultados muestran en la subdimensión docencia mayores porcentajes en los niveles muy alto y alto $(65,28 \%)$, mientras que en las funciones gestión académica e investigación, la mayoría se encuentra entre moderado o bajo $(56,48 \%$ y $68,52 \%$ respectivamente).

Tabla 4

Distribución relativa del nivel de logro de los objetivos institucionales por función sustantiva

\begin{tabular}{lccc}
\hline \multirow{2}{*}{$\begin{array}{c}\text { Nivel de logro } \\
\text { de los objetivos }\end{array}$} & \multicolumn{3}{c}{ Función sustantiva } \\
\cline { 2 - 4 } institucionales & Docencia & $\begin{array}{c}\text { Gestión } \\
\text { académica }\end{array}$ & Investigación \\
\hline Bajo & $3,70 \%$ & $18,98 \%$ & $30,09 \%$ \\
Moderado & $31,02 \%$ & $37,50 \%$ & $38,43 \%$ \\
Alto & $27,78 \%$ & $22,22 \%$ & $16,20 \%$ \\
Muy alto & $37,50 \%$ & $21,30 \%$ & $15,28 \%$ \\
\hline
\end{tabular}

Fuente: Elaboración propia (2019)

Como lo indica el p-valor de la tabla 5, los niveles de control interno en el desempeño docente y el nivel de logro de los objetivos institucionales no tienen relación significativa. Se destacan otros resultados relevantes: el 43,06\% de los docentes presentaron un nivel de desempeño destacado y nivel moderado de logro de los objetivos institucionales; 6,94\% nivel de desempeño destacado y bajo nivel de logro de los objetivos institucionales; 5,09\% nivel de desempeño competente y nivel de logro moderado de objetivos institucionales; $0,93 \%$ nivel de desempeño competente y bajo nivel de logro de objetivos institucionales. Estas cuatro combinaciones representan que el 56,02\% del total de docentes que presentan nivel de desempeño competente o destacado, presentan logros moderados y bajos de objetivos institucionales.

Del análisis estadístico realizado se puede inferir que como el p valor es de $0,232(23,2 \%)$ mayor que el nivel de significancia (5\%) se acepta la hipótesis nula por lo que se puede concluir que el control interno del desempeño docente no se relaciona con el logro de los objetivos institucionales, mejor dicho, las tareas académicas y de investigación de

Tabla 5

Relación entre nivel de desempeño del talento humano docente UTM y logro de objetivos institucionales

\begin{tabular}{|c|c|c|c|c|c|}
\hline \multirow{3}{*}{ Nivel de logro de los objetivos institucionales } & \multicolumn{3}{|c|}{ Nivel de desempeño del talento humano docente } & \multirow{3}{*}{$\mathbf{X}^{2}$} & \multirow{3}{*}{ p-valor } \\
\hline & $\begin{array}{c}\text { Satisfactorio } \\
(60-80)\end{array}$ & $\begin{array}{c}\text { Competente } \\
(81-90)\end{array}$ & $\begin{array}{c}\text { Destacado } \\
(91-100)\end{array}$ & & \\
\hline & n (\%) & n (\%) & n (\%) & & \\
\hline Bajo $(<60)$ & $0(0,00)$ & $2(0,93)$ & $15(6,94)$ & \multirow{4}{*}{8,09} & \multirow{4}{*}{0,232} \\
\hline Moderado $(60-80)$ & $0(0,00)$ & $11(5,09)$ & $93(43,06)$ & & \\
\hline Alto $(81-90)$ & $3(1,39)$ & $6(2,78)$ & $44(20,37)$ & & \\
\hline Muy alto $(91-100)$ & $1(0,46)$ & $2(0,92)$ & $39(18,06)$ & & \\
\hline
\end{tabular}

Nota: prueba de independencia basada en el estadístico chi-cuadrado y razón de verosimilitudes

Fuente: Elaboración propia (2019) 
Tabla 6

Perfil correlativo de R de Pearson: Desempeño del talento humano docente / logro de los objetivos institucionales

\begin{tabular}{llrr}
\hline & & Logro de objetivos & Desempeño docente \\
\hline & Correlación de Pearson & 1 & 0,846 \\
& Sig. (bilateral) & 0,154 \\
Logro de Objetivos & Suma de cuadrados y productos vectoriales & 32747,827 & 152,990 \\
& Covarianza & 152,315 & 0,712 \\
& Muestra & 216 & 216 \\
& Correlación de Pearson & 0,846 & 1 \\
& Sig. (bilateral) & 0,014 & 3847,040 \\
& Suma de cuadrados y productos vectoriales & 152,990 & 17,893 \\
& Covarianza & 0,712 & 216 \\
\hline
\end{tabular}

* La correlación es significativa al nivel 0,05 (bilateral).

los docentes no se han reflejado en los logros que pueda tener la Universidad Técnica de Manabí. Entonces debido a los resultados obtenidos, es necesario implementar políticas que implique corregir esta deficiencia estructural.

\section{DISCUSIÓN}

El estudio revela que no existe relación entre el control interno en el desempeño docente y el logro de objetivos institucionales en la Universidad Técnica de Manabí. Esto se ve reflejado en los docentes que cumplen sus actividades sustantivas sin tener pleno conocimiento de los objetivos institucionales, lo cual es producto de la escasa difusión de los objetivos por parte de las autoridades competentes. En consecuencia, como Alles (2019) sostiene, los objetivos propuestos para el período a evaluar deben ser informados de manera oportuna a todos los actores que participan en la evaluación. Otro factor importante es el insuficiente compromiso institucional de un número representativo de profesores, puesto que un buen docente está al frente de todo desarrollo universitario (Homer, 2013). El análisis estadístico revela que en la UTM no necesariamente los docentes evaluados como destacados contribuyen a niveles altos de logro de objetivos institucionales. Esto es resultado de la escasa cultura de objetividad de los evaluadores, quienes se basaron en criterios de subjetividad, tales como: compañerismo, temor y solidaridad por la estabilidad laboral del evaluado (dos evaluaciones consecutivas con calificaciones inferiores a 60 puntos o cuatro evaluaciones inferiores a 60 puntos durante su carrera son razones para remover o cesar a un docente de la UTM). Esto coincide con las teorías de Alles (2017) y Almuiñas y Galarza (2013) quienes sostienen que las personas que pueden evaluar libremente tienden a ser más sinceros y francos en su rol de evaluador, sin temor a posibles reper- cusiones no deseadas. Al respecto, Tejedor y Jornet (2008) afirman que la evaluación de desempeño docente debe proporcionar información confiable sobre nivel en que los colaboradores contribuyen al logro de los objetivos institucionales. Los resultados del estudio evidenciaron que pese a existir un alto porcentaje de docentes ubicados en la categoría destacados según la percepción de los encuestados el logro de objetivos institucionales se encuentra en su mayoría en el nivel moderado y no en niveles altos o muy altos como se esperaría estar con docentes ubicados en casi su totalidad en la categoría de evaluación integral de desempeño docente.

\section{REFERENCIAS BIBLIOGRÁFICAS}

Alles, M. (2012). Diccionario de términos: Recursos humanos. Buenos Aires: Granica.

Alles, M. (2017). Desempeño por competencias: Estrategia, Desarrollo de personas, Evaluación de $360^{\circ}$. Buenos Aires: Granica.

Alles, M. (2019). Dirección estratégica de RRHH: Gestión por competencias. Buenos Aires: Granica.

Almuiñas, J., \& Galarza, J. (2013). La evaluación del desempeño del docente universitario: experiencias institucionales y nacionales. Guayaquil: Senefelder.

Asamblea Nacional Constituyente de la República del Ecuador (2008). Constitución de la República de la Ecuador. Quito: Asamblea Nacional de la República del Ecuador. Recuperado de https://www.asambleanacional.gob.ec/sites/default/files/constituciondelarepublicadelecuador-incluyereformas-consultapopular7demayo.pdf 
Bohlander, G., Snell, S., \& Morris, S. (2018). Administración de Recursos Humanos. México D.F.: Cengage Learning.

Chiavenato, I. (2017). Administración de recursos humanos: El capital humano de las organizaciones. México D.F.: Mc Graw Hill.

Consejo de Educación Superior (2010). Ley Orgánica de Educación Superior. Ecuador: Sistema de Información de tendencias educativas en América Latina. Recuperado de http://www.siteal.iipe.unesco.org/sites/default/files/sit_accion_files/ec_6011.pdf

Consejo de Educación Superior (2018). Ley Orgánica de Educación Superior. Ecuador: Lexis Finder. Recuperado de http://www.ces.gob.ec/documentos/Normativa/ LOES.pdf

Consejo de Educación Superior (2018). Reglamento de Carrera y Escalafón del Profesor e Investigador del Sistema de Educación Superior. Ecuador: Consejo de Educación Superior. Recuperado de http://gaceta.ces.gob.ec/ resultados.html?id_documento $=195113$

Dessler, G., \& Varela, R. (2017). Administración de recursos humanos: Un enfoque latinoamericano. México D.F.: Pearson.

Fernandes, D., Sotolongo, M., \& Martínez, C. (2016). La Evaluación del Desempeño por Competencias: Per- cepciones de Docentes y Estudiantes en la Educación Superior. Formación universitaria, 9(5), 15-24.

Homer, C. (2013). El surgimiento de las universidades. Buenos Aires: Universidad de Palermo.

Ronquillo, S., Moreira, C., \& Verdesoto, O. (2016). La evaluación docente una propuesta para el cambio en la Facultad de Administración de la universidad ecuatoriana. Revista Universidad y Sociedad, 8(2), 125-131. Recuperado de http://scielo.sld.cu/scielo.php?scrip$\mathrm{t}=$ sci_arttext\&pid=S2218-36202016000200015

Tejedor, F., \& Jornet, J. (2008). La evaluación del profesorado universitario en España. Revista electrónica de investigación educativa, 10(1), 1-29. Recuperado de http://www.scielo.org.mx/scielo.php?script=sci_arttext\&pid=S1607-40412008000300005

Universidad Técnica de Manabí. (2017). Reglamento de evaluación Integral al desempeño del personal académico de la UTM. Recuperado de: https://www.utm. edu.ec/la-universidad/reglamentos/file/14-reglamento-de-evaluacion-integral-al-desempeno-del-personal-academico

Weihrich, H., Cannice, M., \& Koontz, H. (2017). Administración: Una perspectiva global, empresarial y de innovación. México D.F.: Mc Graw Hill Education. 
